\title{
An IGA Method for Magnetostatic Problems Defined on Overlapped Patches
}

\author{
Honghai Zhang, Rong Mo and Neng Wan \\ Key Laboratory of Contemporary Design and Integrated Manufacturing Technology, Ministry of Education, Northwestern \\ Polytechnical University, Xi’an, China, 710072
}

\begin{abstract}
If the union operator is applied in the CAD process, the overlapped parts should be trimmed to carry out IGA(IsoGeometric Analysis), and the integration schemes to keep the integration precision on the trimmed patches are time consuming. In this paper, an IIPG (incomplete interior penalty method) is introduced to IGA to solve the magnetostatic problems which are defined on the overlapped domains. With this method, the time-consuming integration processes on the trimmed patches are avoided.
\end{abstract}

Keywords-isogeometric analysis; discontinuous galerkin method; overlapped domain; magnetostatic

\section{INTRODUCTION}

To obtain an optimized design, the CAD-FEA (computer aided design and finite element analysis) iteration process is often applied in engineering works. In the iteration, researchers often translate the CAD files into analysis-suitable geometries (ASGs) and create the mesh files from the ASGs for the FEA codes. These two tasks take over $80 \%$ of the overall analysis time in the CAD-FEA iteration process. Now, the Isogeometric Analysis (IGA ,Hughes et al.[1]-[4]) is developed to eliminate the gap between the CAD and FEA. With IGA, the refined geometries are kept unchanged exactly[4], [5], and it is efficient to realize h-, p-, k- refinements. In the CAD modeling process, if the union operator is applied to create new CAD models, some of the IGA methods[6]-[8] need to concern the integration precision on the trimmed parts, because the Boolean operations (union, subtraction and intersection) are based on the trimming algorithms. Usually, the integration schemes on the trimmed patches are time consuming. Recently, other IGA methods, for example he Additive Schwarz Domain Decomposition Method (ASDDM [9]) and IGAODG[10] are developed, and these methods avoid the integrations on the trimmed patches when the union operation is applied. This paper follows the works of [10] and presents an IGA IIPG method to solve the magnetostatic problems on the overlapped domains.

The paper is arranged as follows. In section II, we briefly introduce some requisite notations for overlapped patches. In section III, we create the discretization scheme for the magnetostatic equations. In section IV, the convergence property is tested numerically. Finally, the paper is summarized in section $\mathrm{V}$.

\section{IGA AND SOME NOTATIONS}

\section{A. On a Single Patch}

In the following discussions, the hat ' $\wedge$ ' is used to indicate that a function or a space is defined on the parametric domain. Between the parametric patch $\hat{P}$ and the physical patch $P$, a NURBS-based geometry can be seen as a map $F: \hat{P} \rightarrow P$. Therefore, a discrete function space on the physical patch can be defined with

$$
S^{h}(P):=\operatorname{span}\left\{\hat{B}_{i} \circ F^{-1}\right\},
$$

where $\hat{B}_{i}$ is the $i$-th NURBS basis. On a parametric patch, the open knot vectors indicate a mesh, marked as $M_{p r}(\hat{P})$, and a physical mesh $M_{p r}(P)$ can be defined by

$$
M_{p r}(P)=\left\{K \mid K=F(\hat{K}), \hat{K} \in M_{p r}(\hat{P})\right\} .
$$

Defining a function $\delta_{K}$ by

$$
\delta_{K}=\left\{\begin{array}{l}
0, x \notin K \\
1, \quad \text { else }
\end{array},\right.
$$

the space $S^{h}(P)$ can be further broken into a space $S_{K}^{h}(P)$ where

$$
S_{K}^{h}(P):=\operatorname{span}\left\{v_{K}^{h} \mid v_{K}^{h}=\delta_{K} v^{h}, \forall v^{h} \in S^{h}(P)\right\} .
$$

Functions in $S_{K}^{h}(P)$ may be non-zero out of an element $K$. We use the definition (2) to avoid discussing different continuities of basis between elements.

\section{B. On United Patches}

We suppose that a parametric patch $\hat{P}$ is precisely meshed into a collection of elements $M_{p r}(\hat{P})=\{\hat{K}\}$. If the CAD model contains multiple patches, we denote by $\hat{T}_{h}=\bigcup_{i} M_{p r}\left(\hat{P}_{i}\right)$ the 
parametric mesh. Similarly, the physical mesh is defined by $T_{h}=\bigcup_{i} M_{p r}\left(P_{i}\right)$ where

$$
M_{p r}(P)=\left\{K \mid K=F(\hat{K}), \quad \hat{K} \in M_{p r}(\hat{P})\right\} .
$$

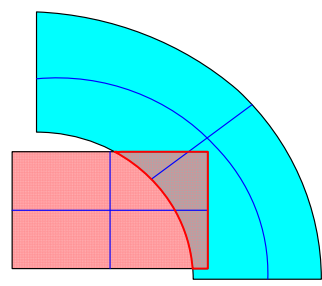

FIGURE I.

$$
\text { TWO UNITED PATCHES. }
$$

We also suppose that the meshes $T_{h}$ and $\hat{T}_{h}$ are quasiuniform, so that we can use a single symbol $h$ to represents the sizes of both meshes.

If the patches are overlapped, we need three kinds of boundary collections as defined in(3). The first collection $\Gamma_{d}$ contains the external boundaries of the whole domain $\Omega$. The second collection $\Gamma_{e}$ contains all the adjacent boundaries of brother elements in their parent patches. The third collection $\Gamma_{p}$ contains all the external boundaries of patches, which do not belong to $\Gamma_{e} U \Gamma_{d}$. In Figure II, boundaries colored red $\subset \Gamma_{p}$, boundaries colored blue $\subset \Gamma_{e}$, and boundaries colored black $\subset \Gamma_{d} \quad$.Finally, we denote by $\Gamma:=\Gamma_{d} \cup \Gamma_{e} \cup \Gamma_{p}=\bigcup_{K}(\partial K)$ the collection of all boundaries of all elements.

$$
\begin{aligned}
& \Gamma_{d}:=\bigcup_{K \in T_{h}}(\partial \Omega \cap \partial K) \\
& \Gamma_{e}:=\bigcup_{P}\left\{e \mid e=\partial K_{i} \cap \partial K_{j}, \forall K_{i}, K_{j} \in M_{p r}(\mathrm{P}), i \neq j\right\} \\
& \Gamma_{p}:=\bigcup_{K}(\partial K) \backslash\left(\Gamma_{e} \bigcup \Gamma_{d}\right)
\end{aligned}
$$

On the overlapped mesh, we define space $H^{k}\left(T_{h}\right)$ as follows: 1) a function $u$ on each element $K \in T_{h}$ belongs to $\left.H^{k}(K), 2\right)$ the function $u_{K}$ needs not to be continuous with its brother elements, and 3) the function $u_{K}$ needs not to be as same as the fields on the other overlapped elements of other parent patches. On $H^{k}\left(T_{h}\right)$, the Sobolev norm is defined by the $L^{2}$ inner product as

$$
\|u\|_{k, T_{h}}^{2}=\sum_{K \in T_{h}}\left\|u_{K}\right\|_{k, K}^{2}, \quad\left\|u_{K}\right\|_{k, K}^{2}:=\sum_{i=0}^{k}<D^{i} u, D^{i} u>_{L^{2}(K)} .
$$

Similarly, we define a discrete space $S_{K}^{h}\left(T_{h}\right) \subset H^{k}\left(T_{h}\right)$ by

$$
S_{K}^{h}\left(T_{h}\right)=\operatorname{span}\left\{v_{K}^{h} \mid v_{K}^{h}=\delta_{K} v^{h}, \forall v^{h} \in \forall S^{h}\left(P_{i}\right), \bigcup P_{i}=\Omega\right\}
$$

On the boundary $\partial K_{i}$, for a scalar function $v_{K_{i}} \in S_{K}^{h}\left(T_{h}\right)$, we define its adjacent value $v_{K_{i}, e}$ by

$$
v_{K_{i}, e}(x)=\left\{\begin{array}{l}
v_{K_{j}}(x), \quad \text { on } e \subset \Gamma_{e}, \text { if } x \in \partial K_{i} \cap \partial K_{j} \\
0, \quad \text { on } e \subset \Gamma_{d} \\
v_{K_{j}}(x), \quad \text { on } e \subset \Gamma_{p}, \text { if } x \in \partial K_{i} \cap K_{j}
\end{array} .\right.
$$

If no ambiguity exists, we directly reuse the symbol $S_{K}^{h}\left(T_{h}\right)$ to represent the discrete space of vector (tensor) functions and reuse $v_{K_{i}, e}(x)$ to represent the adjacent value of a vector (tensor) functions.

\section{DISCRETIZATION SCHEME}

A model equation of the magnetostatic problem reads as

$$
\begin{aligned}
& \nabla \times \nabla \times A=f, \quad \text { in } \Omega \\
& A=0, \quad \text { on } \partial \Omega
\end{aligned},
$$

where $A$ is the vector potential. Under the definition of the jump operator

$$
\begin{aligned}
& {[A]=n \times\left(A_{1}-A_{2}\right), \quad \text { on } \Gamma \backslash \Gamma_{d},} \\
& {[A]=n \times A, \quad \text { on } \Gamma_{d}}
\end{aligned}
$$

the IIPG for non-overlapped patches reads as:

Find $A^{h} \in S_{K}^{h}\left(T_{h}\right)$, such that

$$
\begin{gathered}
\int_{T_{h}} v^{h} \cdot f=\int_{T_{h}}\left(\nabla \times A^{h}\right) \cdot\left(\nabla \times v^{h}\right) \\
-\sum_{e \in \Gamma} \int_{e}\left\{\nabla \times A^{h}\right\} \cdot\left[v^{h}\right] d s+\int_{e} \alpha\left[A^{h}\right] \cdot\left[v^{h}\right] d s . \\
, \quad \forall v^{h} \in S_{K}^{h}\left(T_{h}\right)
\end{gathered}
$$

For two vector $A$ and $V$, a computation under the Green formula gives

$$
\begin{gathered}
\int_{\Omega} V \cdot(\nabla \times \nabla \times A) d x=\int_{\Omega}(\nabla \times A) \cdot(\nabla \times V) d x \\
-\int_{\partial \Omega}(n \times V)(\nabla \times A) d s,
\end{gathered}
$$

and this can be seen as a formula of the integration by parts. For the second item of (8), we have 


$$
\begin{aligned}
-\int_{e}\{\nabla \times A\} \cdot n \times\left(v_{1}-v_{2}\right) d s & \\
=-\int_{e} & \frac{\nabla \times A_{1}+\nabla \times A_{2}}{2} \cdot n \times\left(v_{1}-v_{2}\right) d s \\
\stackrel{v_{2}=0}{=} \int_{e}( & \left.-\nabla \times A_{1}\right) \cdot\left(n \times v_{1}\right) d s \\
& \quad+\int_{e}\left(\frac{\nabla \times A_{1}-\nabla \times A_{2}}{2}\right) \cdot n \times v_{1} d s
\end{aligned}
$$

From(9), it can be seen that $\left(\nabla \times A_{1}-\nabla \times A_{2}\right)$ is penalized by $\frac{n \times v_{1}}{2}$ on a boundary, and $\int_{e}\left(-\nabla \times A_{1}\right) \cdot\left(n \times v_{1}\right) d s$ is a component of the formula of the integration by parts. Thus, when extending the $\mathrm{C}^{0}$ - and $\mathrm{C}^{1}$ - punishments onto $\Gamma_{p}$ with the coefficients deduced from (8), the IGA IIPG for overlapped domain reads as:

Find $A^{h} \in S_{K}^{h}\left(T_{h}\right)$ such that

$$
\begin{aligned}
\int_{T_{h}} v_{K}^{h} \cdot f d x & =\int_{T_{h}}\left(\nabla \times A_{K}^{h}\right) \cdot\left(\nabla \times v_{K}^{h}\right) d x \\
& -\sum_{K \in T_{h}} \int_{\partial K} \nabla \times A_{K}^{h} \cdot\left(n \times v_{K}^{h}\right) d s \\
+ & \sum_{K \in T_{h}} \int_{\partial K \cap\left(\Gamma \backslash \Gamma_{d}\right)}\left(\nabla \times A_{K}^{h}-\nabla \times A_{K, e}^{h}\right) \cdot \frac{n \times v_{K}^{h}}{2} d s \\
+ & \sum_{K \in T_{h}} \int_{\partial K} \alpha_{e} n \times\left(A_{K}^{h}-A_{K, e}^{h}\right) \cdot\left(n \times v_{K}^{h}\right) d s, \quad \forall v_{K}^{h} \in S_{K}^{h}\left(T_{h}\right)
\end{aligned}
$$

In(10), the divergence of the vector potential is not penalized, and $S_{K}^{h}\left(T_{h}\right)$ is not divergence-free (see [11], [12] and their references). Therefore, in the vector $2 \mathrm{D}$ situation, the divergence-free condition is applied to each of the elements by adding the item $\sum_{K \in T_{h}} \int_{K}\left(\nabla \cdot A_{K}^{h}\right)\left(\nabla \cdot v_{K}^{h}\right) d x$ to the left side of(10).

\section{NUMERICAL EXAMPLES}

\section{A. For 1D Cases}

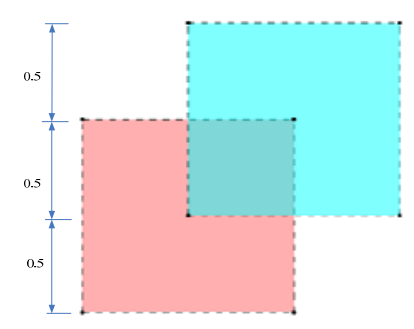

FIGURE II.

THE UNION OF TWO OVERLAPPED SQUARES

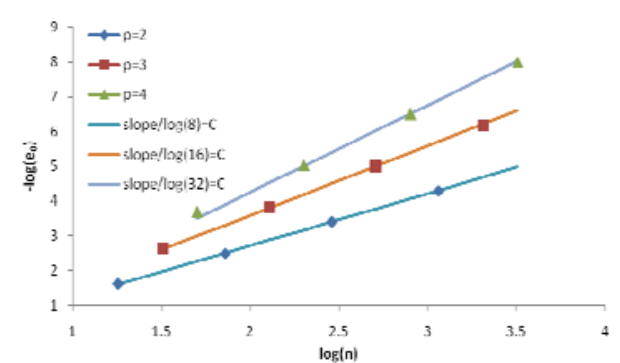

(A) CONVERGENCE PROPERTIES ON $\left\|u-u^{h}\right\|_{0, T_{h}}$

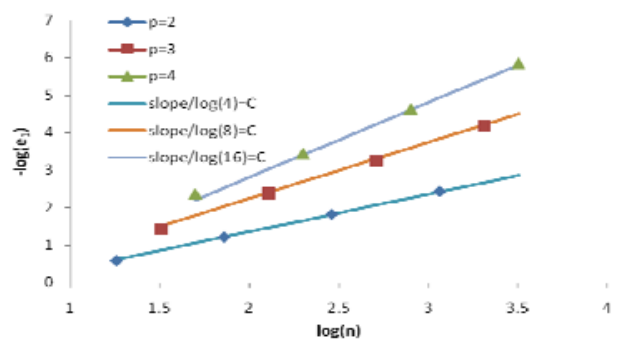

(B) CONVERGENCE PROPERTIES ON $\left\|u-u^{h}\right\|_{1, T_{h}}$

FIGURE III.

CONVERGENCE PROPERTIES OF 1D-PROBLEM

For the vector-1D problem (11) defined on the domain shown in Figure II, the convergence properties is given in Figure III.

$$
\begin{aligned}
& \nabla \times \nabla \times A=-2 \cos \left(\frac{x^{2}+y^{2}}{2}\right)+\left(x^{2}+y^{2}\right) \sin \left(\frac{x^{2}+y^{2}}{2}\right) \\
& \left.A\right|_{\partial \Omega}=\sin \left(\frac{x^{2}+y^{2}}{2}\right)
\end{aligned}
$$

In the figures, $n$ denotes the variable numbers, and $e_{i}$ denotes the error $\left\|u-u^{h}\right\|_{i, T_{h}}$.

From Figure III, it is observed that when the polynomial order varies from 2 to 4 , the convergence speeds are optimal with respect to the norm $\|\cdot\|_{0, T_{h}}$ and $\|\cdot\|_{1, T_{h}}$.

\section{B. For 2D Cases}

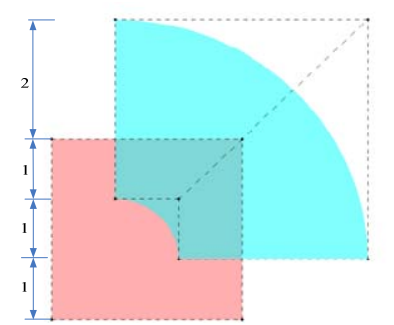

(A)

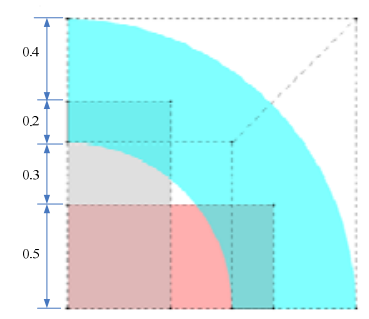

(B)
FIGURE IV. TWO OVERLAPPED DOMAINS. (A) WITH TWO PATCHES, (B) WITH THREE PATCHES 


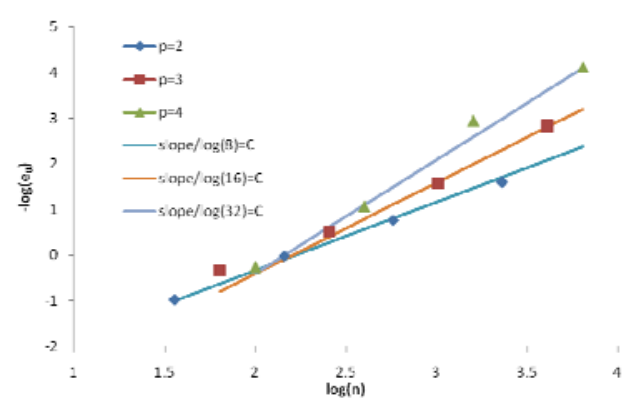

(A) CONVERGENCE PROPERTIES ON $\left\|u-u^{h}\right\|_{0, T_{h}}$

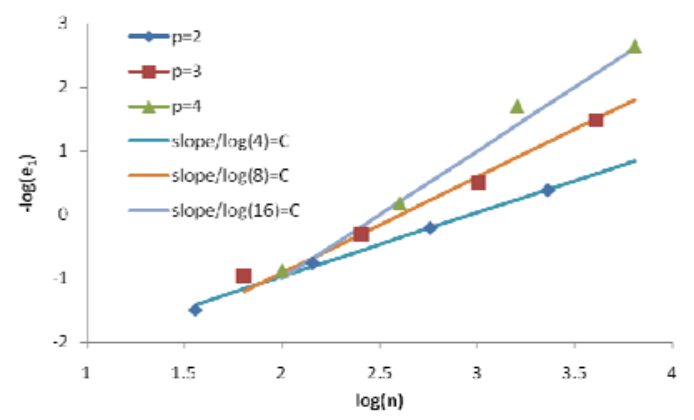

(B) CONVERGENCE PROPERTIES ON $\left\|u-u^{h}\right\|_{1, T_{h}}$

FIGURE V. CONVERGENCE PROPERTIES FOR FIGURE IV(A)

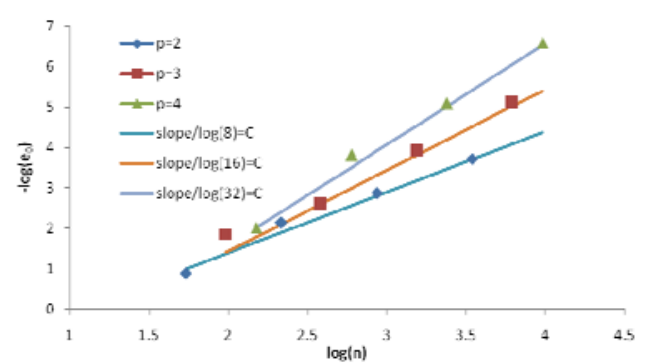

(A) CONVERGENCE PROPERTIES ON $\left\|u-u^{h}\right\|_{0, T_{h}}$

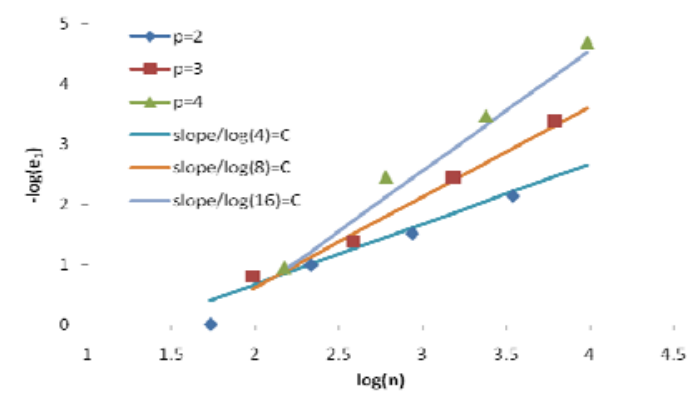

(B) CONVERGENCE PROPERTIES ON $\left\|u-u^{h}\right\|_{1, T_{h}}$

FIGURE VI. CONVERGENCE PROPERTIES FOR FIGURE IV(B)

For the problem(12) defined on the domains in Figure IV, the convergence properties are listed in Figures V and VI respectively.

$$
\begin{aligned}
& \nabla \times \nabla \times A=\left(\begin{array}{l}
2 \sin (x) \cos (y) \\
-2 \cos (x) \sin (y)
\end{array}\right) \\
& \left.A\right|_{\partial \Omega}=\left(\begin{array}{l}
\sin (x) \cos (y) \\
-\cos (x) \sin (y)
\end{array}\right)
\end{aligned}
$$

From Figures V and VI, the same optimal convergence orders are obtained as shown in Figure III.

\section{SUMMARY}

Firstly, the IIPG for the magnetostatic problems is discussed in the non-overlapped cases. Secondly, by applying the deduced punishing coefficients on the overlapped boundaries, the IGA IIPG for overlapped domains is created to solve the magnetostatic problems. In the $1 \mathrm{D}$ and $2 \mathrm{D}$ cases, the numerical results show that the presented method can reach the optimal convergence orders when the patches are overlapped. It is also found that, the classical method to impose the divergence-free condition is available in the presented method.

\section{ACKNOWLEDGMENT}

The project is supported by the National Natural Science Fund (Program No.51775445) and the Natural Science Basic Research Plan in Shaanxi Province of China (Program No.2016JM5040).

\section{REFERENCES}

[1] Y. Bazilevs, L. Beirão Da Veiga, J. A. Cottrell, T. J. R. Hughes, And G. Sangalli, "Isogeometric Analysis: Approximation, Stability And Error Estimates For H-Refined Meshes,” Math. Model. Methods Appl. Sci., Vol. 16, No. 07, Pp. 1031-1090, 2006.

[2] J. A. Cottrell, T. J. R. Hughes, And Y. Bazilevs, Isogeometric Analysis Toward Intergration Of Cad And Fea. A John Wiley And Sons, Ltd., 2009.

[3] T. J. R. Hughes, J. A. Cottrell, And Y. Bazilevs, "Isogeometric Analysis: Cad, Finite Elements, Nurbs, Exact Geometry And Mesh Refinement," Comput. Methods Appl. Mech. Eng., Vol. 194, No. 39-41, Pp. 41354195, 2005.

[4] L. Beirão Da Veiga, A. Buffa, J. Rivas, And G. Sangalli, "Some Estimates For H-P-K-Refinement In Isogeometric Analysis,” Numer. Math., Vol. 118, No. 2, Pp. 271-305, Oct. 2010.

[5] J. Cottrell, T. J. R. Hughes, And A. Reali, "Studies Of Refinement And Continuity In Isogemetric Analysis,” Comput. Methods Appl. Mech. Eng., Vol. 196, Pp. 4160-4183, 2007.

[6] D. Natekar, X. Zhang, And G. Subbarayan, "Constructive Solid Analysis: A Hierarchical, Geometry-Based Meshless Analysis Procedure For Integrated Design And Analysis," Cad Comput. Aided Des., Vol. 36, No. 5, Pp. 473-486, 2004.

[7] B.-Q. Zuo, Z.-D. Huang, Y.-W. Wang, And Z.-J. Wu, "Isogeometric Analysis For Csg Models,” Comput. Methods Appl. Mech. Engrg., Vol. 285, Pp. 102-124, 2015.

[8] Y. Wang, Z. Huang, Y. Zheng, And S. Zhang, "Isogeometric Analysis For Compound B-Spline Surfaces,” Comput. Methods Appl. Mech. Eng., Vol. 261-262, Pp. 1-15, 2013.

[9] M. Bercovier And I. Soloveichik, “Overlapping Non Matching Meshes Domain Decomposition Method In Isogeometric Analysis,” Arxiv Prepr. Arxiv1502.03756, 2015.

[10] H. Zhang, R. Mo, And N. Wan, “An Iga Discontinuous Galerkin Method On The Union Of Overlapped Patches,” Comput. Methods Appl. Mech. Eng., Vol. 326, Pp. 446-480, 2017. 
[11] B. Cockburn, F. Li, And C.-W. Shu, "Locally Divergence-Free Discontinuous Galerkin Methods For The Maxwell Equations," $J$. Comput. Phys., Vol. 194, No. 2, Pp. 588-610, 2004.

[12] M. Costabel And M. Dauge, "Weighted Regularization Of Maxwell Equations In Polyhedral Domains,” Numer. Math., Vol. 93, No. 2, Pp. 239-277, Dec. 2002. 